\title{
Symmetry-breaking-induced rare fluctuations in a time-delay dynamic system
}

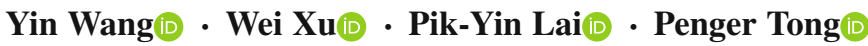

Received: 20 June 2020 / Accepted: 20 February 2021 / Published online: 6 March 2021

(C) The Author(s), under exclusive licence to Springer Nature B.V. part of Springer Nature 2021

\begin{abstract}
Inspired by the experimental and numerical findings, we study the dynamic instabilities of two coupled nonlinear delay differential equations that are used to describe the coherent oscillations between the top and bottom boundary layers in turbulent RayleighBénard convection. By introducing two sensitivity parameters for the instabilities of the top and bottom boundary layers, we find three different types of solutions, namely in-phase single-period oscillations, multi-period oscillations and chaos. The chaos solution contains rare but large amplitude fluctuations. The statistical properties of these fluctuations are consistent with those observed in the experiment for the massive eruption of thermal plumes, which causes random reversals of the large-scale circulation in turbulent Rayleigh-Bénard convection. Our study thus provides new insights into the origin of rare massive eruptions and sudden changes of large-scale flow pattern that are often observed in convection systems of geophysical and astrophysical scales.
\end{abstract}

\section{Y. Wang $\cdot$ W. Xu $\cdot$ P. Tong $(\varangle)$}

Department of Physics, Hong Kong University of Science and Technology, Clear Water Bay, Kowloon, Hong Kong e-mail: penger@ust.hk

Y. Wang

Princeton Plasma Physics Laboratory, Princeton

University, Princeton, NJ 08543, USA

P.-Y. Lai

Department of Physics and Center for Complex Systems, National Central University, Chungli 320, Taiwan, R.O.C.
Keywords Symmetry breaking - Delay differential equations · Turbulent thermal convection - Random reversal $\cdot$ Bifurcation

\section{Introduction}

As a classical model system, Rayleigh-Bénard convection (RBC) has been used for the study of a range of hydrodynamic problems over a hundred years. It consists of a fluid layer of thickness $H$, which is heated at the bottom and cooled from the top with a constant temperature gradient parallel to gravity. Once the temperature drop $\Delta T$ across the fluid layer, or equivalently the Rayleigh number $\mathrm{Ra}$ (dimensionless buoyancy), is larger than a critical value $\operatorname{Ra}_{c}(\simeq 1708)$, the bulk fluid becomes unstable and convection sets in $[20,26]$. From the onset of convection up to $\mathrm{Ra} \sim 10 \mathrm{Ra}_{c}$, periodic flow structures form in the horizontal plane and cellular patterns can be observed from above [16,23]. With further increase in Ra, the number of characteristic frequencies increases and the flow steps into a chaotic regime [36,47]. As a well-controlled hydrodynamic system, RBC has become a paradigm for the development of stability theory in hydrodynamics $[20,26]$ and for our understanding of pattern formation and spatialtemporal chaos $[8,23,33]$.

For even larger values of $\mathrm{Ra}\left(\gtrsim 10^{8}\right)$, a large-scale circulation (LSC) emerges with a size comparable to the convection cell $[18,41]$. Driven by the thermal plumes emitted from the unstable thermal boundary 
layers near the conducting plates, the LSC remains as a steady state in a turbulent background $[28,53,69,79]$. Many studies of the LSC have been carried in upright cylindrical cells of aspect ratio unity, in which the LSC has a single roll structure occupying the bulk of the convection cell. An intriguing feature associated with the LSC is the observation of a well-defined oscillation in the temperature [18,60] and velocity [55] power spectra at large $\mathrm{Ra}$. The synchronized oscillations are related to the dynamics of the LSC [31,52,54,56,81]. Theoretical efforts were made to explain the origin of the coherent oscillations, based on either stochastic or deterministic differential equations $[14,15,39,72]$. For upright cylinders, rectangular and cubic cells, the coherent oscillations were explained by the torsional [31] and sloshing [81] modes of the LSC, resulting from a resonant oscillation of the LSC in a potential well owing to the geometric confinement of the LSC in a closed convection cell $[14,15]$.

Recently, a novel convection experiment was conducted in a thin disk cell, whose circular plane is orientated vertically parallel to gravity $[65,73,75]$. Owing to its circular cross section that matches the single-roll structure of the LSC, other secondary flow modes, such as sloshing and torsional oscillations, cannot be excited in this quasi-two-dimensional (2D) system. Even in such a simple quasi-2D system, strong in-phase oscillations were found in the velocity and temperature time series data obtained at two opposite positions in the midplane of the cell. This coherent oscillation is well described by the deterministic model proposed by Villermaux $[65,72]$. In this model, the boundary layer instabilities are triggered by the incoming thermal plumes transported by the LSC. Consequently, the thermal plumes are emitted simultaneously from the two boundary layers with a local frequency $f_{0} \simeq U_{0} /(2 H)$, where $U_{0}$ is the velocity of LSC, and thus, $2 H / U_{0}$ is the turnover time of the LSC.

Another interesting behavior of the LSC is its irregular cessation, which is a momentary breakdown of the entire circulation flow followed by a newly formed LSC in a randomly chosen direction $[12,21,66,80]$. It is called cessation if the rotational direction of the new LSC is the same as that of the original LSC. Otherwise, it is called reversal. A number of stochastic or deterministic models have been proposed to explain the observed cessations and reversals of the LSC [5$7,11,13,19,32,50,68]$. Nonetheless, a general understanding of the phenomena has not been reached. So far, most of the experiments devoted to testing the theoretical models were conducted in either rectangular or cylindrical cells. Because the single-roll structure of the LSC does not fit the geometry of these convection cells very well, secondary (corner) flows in the cell cause complications of the LSC dynamics [68]. Consequently, a general mechanism that causes the LSC cessations and reversals has not yet been obtained.

In a more recent experiment [75], we found that random cessations and reversals of the LSC could also be realized and quantitatively studied in the specially designed thin disk cell. This is a "simple but not simpler" system, which retains main features of turbulent convection [65] and provides a useful experimental platform for the study of intrinsic stabilities of the LSC. The experiment clearly showed that the LSC reversals are caused by rare massive eruptions of thermal plumes, which disrupt the existing LSC and reset its rotational direction. It is found that the convective flow is in a special chaotic state, i.e., it contains a steady "quiet period" with a minute amount of heat accumulation for a long time, followed by a short and intermittent "active period" which releases the accumulated heat by triggering a massive eruption of thermal plumes. This finding is against the common belief that the heat flux going through a RBC system is always balanced at any given time so that the flow can be maintained at a steady state. It thus represents a new kind of convective instability for turbulent RBC that may have important geophysical and astrophysical implications.

In thermal convection systems of geophysical and astrophysical scales, one often observes massive eruption events and sudden changes of large-scale flow patterns. Examples include Earth's outer core convection associated with dynamo generation, oceanic circulation and the convective zone of sun and other stars $[3,17,35$, $38,48,57]$. Owing to their complex material parameters and extreme conditions [1], our fundamental understanding of the geophysical- and astrophysical-scale flow phenomena is often challenged by limited experimental observations and oversimplified computer simulations. Turbulent RBC in the thin disk cell thus provides a unique system for the quantitative study of large-scale flow reversals and massive eruption of thermal plumes. It is of particular interest to know the mechanism that triggers the massive eruptions in a closed convection system. Are these eruptions caused by some minute broken symmetry in the boundary conditions, which may generate a small amount of heat accumula- 
tion continuously during the quiet period? Such a heat accumulation can drive the system into a critical state, at which the boundary layers become supersensitive to turbulent disturbances that trigger the massive eruption of thermal plumes. Or are these eruptions simply triggered by some rare turbulent fluctuations with an extremely large amplitude?

In this paper, we report a combined experimental, numerical and theoretical study aimed at understanding the physical mechanism of the LSC reversals and rare massive eruption of thermal plumes in turbulent RBC. In particular, we investigate the dynamic instabilities of the two coupled nonlinear delay differential equations (DDEs, see Eq. (5)) that was used to describe the coherent oscillations between the top and bottom boundary layers $[65,72]$. By introducing two different sensitivity parameters, $\mu_{t} \neq \mu_{b}$, to external perturbations for the instabilities of the top and bottom boundary layers, we study how the broken symmetry of the boundary conditions near the top and bottom conducting plates affects the dynamic instabilities of the system in a broad range of the parameter space. A central finding of this investigation is that with $\mu_{t} \neq \mu_{b}$, we find a new class of chaotic solutions, which contain rare but large amplitude fluctuations, whose statistical properties are consistent with those observed in the experiment for the massive eruption of thermal plumes. The study of the DDEs thus provides new insights into the origin of the LSC reversals and rare massive eruption of thermal plumes.

\section{Experimental and numerical methods}

\subsection{Experiment}

Details about the experimental apparatus and procedures that are used in this experiment have been described elsewhere [65,73-76], and only some key points are mentioned here. The convection cell used is a thin circular disk with a diameter $D=188 \mathrm{~mm}$ and thickness $W=20 \mathrm{~mm}$, which correspond to an aspect ratio $\Gamma \equiv W / D=0.11$. The circular cross section of the cell is aligned parallel to gravity. The top and bottom $1 / 3$ of the circular sidewall are made of 8 $\mathrm{mm}$-thick copper. The remaining $1 / 6$ of the sidewall on either side of the cell is made of 18-mm-thick transparent Plexiglas. The front and rear circular walls of the cell are also made of the same Plexiglas. The bottom conducting plate is heated uniformly using two silicon rubber film heaters. The top plate is kept at a constant temperature by using a cooling circulator with a temperature stability of $10 \mathrm{mK}$. The temperature of the top and bottom plates is measured using calibrated thermistors with an accuracy of $5 \mathrm{mK}$.

The design of the thin disk cell has two advantages. First, it has a quasi-2D circular shape so that 3D flow modes, such as sloshing and torsional oscillations [14, 15,81 , as well as corner flows [68] cannot be excited in this system. Second, the thin disk cell allows us to use a quantitative shadowgraphic technique to visualize the LSC and plume dynamics and to detect the net heat flux accumulation or loss across the entire cell.

In the experiment, the entire convection cell is placed inside a thermostatic box, in which the temperature is maintained at $(40 \pm 0.1)^{\circ} \mathrm{C}$ to match the mean temperature of the bulk fluid, and thus, the heat exchange with the surroundings is minimized. For this system, the Rayleigh number is defined as $\mathrm{Ra} \equiv \alpha g \Delta T D^{3} /(\nu \kappa)$, where $\alpha, v$ and $\kappa$ are, respectively, the thermal expansion coefficient, kinematic viscosity and thermal diffusivity of the convecting fluid, and $g$ is the gravitational acceleration. The temperature difference across the cell is $\Delta T=T_{b}-T_{t}$, where $T_{b}$ and $T_{t}$ are, respectively, the temperature of the bottom heating plate and top cooling plate. The Prandtl number is given by $\operatorname{Pr}=v / \kappa$. In the experiment, the Ra range varied is $3 \times 10^{9} \lesssim R a \lesssim 1.2 \times 10^{10}$ and $\mathrm{Pr}$ is fixed for a given fluid. Three working fluids are used: one is distilled water $(\operatorname{Pr}=4.4)$ and the other two are the $10 \mathrm{wt} \%$ $(\mathrm{Pr}=5.7)$ and $20 \mathrm{wt} \%(\mathrm{Pr}=7.6)$ aqueous solutions of glycerin.

Measurements of the net accumulation or loss of heat flux across the entire cell are made using the same shadowgraphic technique as that described in Ref. [75]. The measured intensity of the shadowgraph image $I(x, y, t)$ is proportional to the second-order spatial derivative (Laplacian) of the refractive index variation $n(x, y, t)$, which in turn is proportional to temperature fluctuations $T(x, y, t)$ for small values of $T(x, y, t)(\lesssim$ 25K) [25,64]. Therefore, we obtained [75]

$\zeta(x, y, t) \equiv \frac{I_{0}(x, y)-I(x, y, t)}{I(x, y, t)}=\xi \delta J(t)$,

where $I_{0}$ is the background intensity, and $\delta J(t) \equiv$ $J_{b}(t)-J_{t}(t)$ is the net heat accumulation with $J_{b}(t)$ and $J_{t}(t)$ being, respectively, the instantaneous heat flowing into the cell from the bottom conducting plate and 
going out of the cell from the top conducting plate. In the above, $\xi$ is an instrument constant, which is proportional to $\gamma=\mathrm{d} n / \mathrm{d} T$. Equation (1) thus provides a sensitive null test on whether the total heat flow $\delta J(t)$ is balanced or not at any given time.

\subsection{Direct numerical simulation}

Details about the numerical code used in the numerical study have been described elsewhere [76], and here we only mention some key points. The governing equations of the direct numerical simulation are the incompressible Navier-Stokes equation and the convective heat equation under the Boussinesq approximation, with

$$
\begin{aligned}
& \hat{\nabla} \cdot \hat{\boldsymbol{u}}=0, \\
& \hat{\boldsymbol{u}}_{\hat{t}}+(\hat{\boldsymbol{u}} \cdot \hat{\nabla}) \hat{\boldsymbol{u}}=-\nabla \hat{p}+\frac{1}{\sqrt{R a / P r}} \hat{\nabla}^{2} \hat{\boldsymbol{u}}+\hat{T}, \\
& \hat{T}_{\hat{t}}+(\hat{\boldsymbol{u}} \cdot \hat{\nabla}) \hat{T}=\frac{1}{\sqrt{R a \cdot P r}} \hat{\nabla}^{2} \hat{T},
\end{aligned}
$$

where the temperature, length and time are normalized, respectively, by the temperature difference $\Delta T$ across the cell, cell diameter $D$ and free-fall time $\sqrt{D /(g \alpha \Delta T)}$. Equation (2) is solved using the opensource code Nek5000 [30], which uses a spectral element method to accurately resolve the gradients in the velocity field $\hat{\boldsymbol{u}}(\boldsymbol{r}, t)$ and temperature field $\hat{T}(\boldsymbol{r}, t)$. More details about the numerical scheme and appropriate grid resolutions can be found elsewhere [24,30,61].

Our direct numerical simulation is carried out at fixed values of $\mathrm{Ra}=5 \times 10^{9}$ and $\mathrm{Pr}=4.4$ in a vertical thin disk having the same dimensions as those used in the experiment. The non-slip boundary conditions are used for the velocity field. For the temperature field, isothermal boundary conditions are used for the bottom and top conducting surfaces and adiabatic boundary conditions are used for the other walls. The primary mesh size and polynomial order within each mesh element are chosen such that we have 8 grid points to resolve the thermal boundary layer, which is the smallest length scale for turbulent $\mathrm{RBC}$ with $\operatorname{Pr}>1$. Totally, there are 16,200 primary mesh elements and about 8.3 million grid points. The direct numerical simulation is run over $1 \mathrm{~h}$ in real time to reach a steady state, followed by another $5.7 \mathrm{~h}$ (15,500 free-fall times) continuing evolution in order to obtain time series data with adequate statistics.
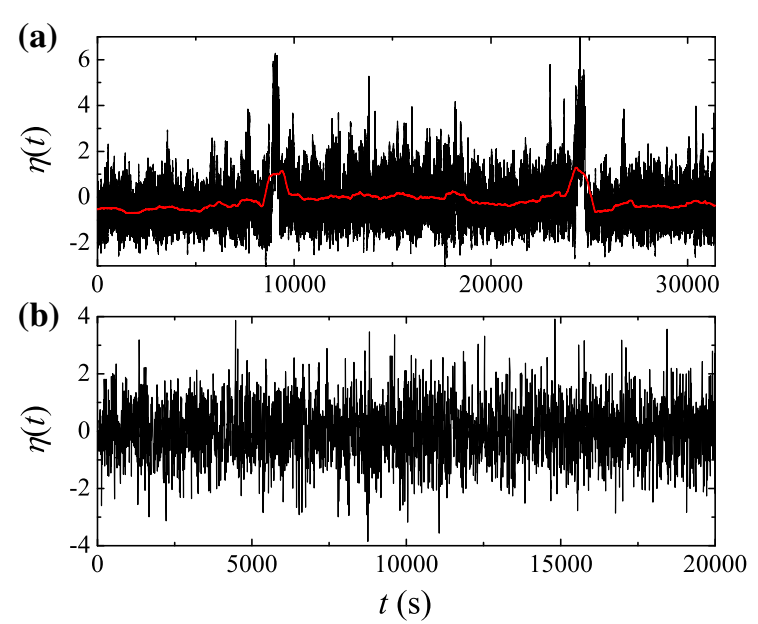

Fig. 1 a Experimentally obtained time series data of the normalized net heat accumulation $\eta(t)$ over a 9-hour-long period of time $t$ (black curve). The red curve shows a moving average of the raw data with a time window of $500 \mathrm{~s}$. The measurements were made at fixed values of $\mathrm{Ra}=6.2 \times 10^{9}$ and $\operatorname{Pr}=4.4$ (adopted from Ref. [75]). b Numerically obtained time series data of $\eta(t)$ from direct numerical simulation at fixed values of $\mathrm{Ra}=5 \times 10^{9}$ and $\operatorname{Pr}=4.4$. (Color figure online)

\section{Numerical and experimental results}

The black curve in Fig. 1a shows the experimentally obtained time series data of the normalized net heat accumulation $\eta(t) \equiv(\delta J(t)-\langle\delta J(t)\rangle) / \sigma_{\delta J}$, over a 9 hour-long period with a sampling frequency of $10 \mathrm{~Hz}$. Here $\langle\delta J(t)\rangle(\simeq 0)$ is the mean value of $\delta J(t)$ and $\sigma_{\delta J}$ is its standard deviation. It is seen that the LSC is in a steady state ("quite period") most of the time so that the measured $\eta(t)$ fluctuates around a constant slightly less than zero. This is seen more clearly from the red curve, which is a moving average of the raw data with a time window of $500 \mathrm{~s}$. A few large spikes are observed in the measured $\eta(t)$ (such as at $t \simeq 9000$ $\mathrm{s}$ and $t \simeq 25,000 \mathrm{~s}$ ), and they are associated with massive eruptions of thermal plumes across the entire cell. These eruption events are rare and last for 1-5 minutes each time ("active period"), during which the existing LSC is destroyed [75]. The measured $\eta(t)$ is positive during the short active period, suggesting that a large amount of heat is released during the massive eruptions. The released heat is accumulated during the long quite period with a small negative mean value of $\eta(t)$.

Figure 1b shows the numerically obtained time series data of $\eta(t)$ from the direct numerical simulation over a 5.7-hour-long period of time $t$. The values of 


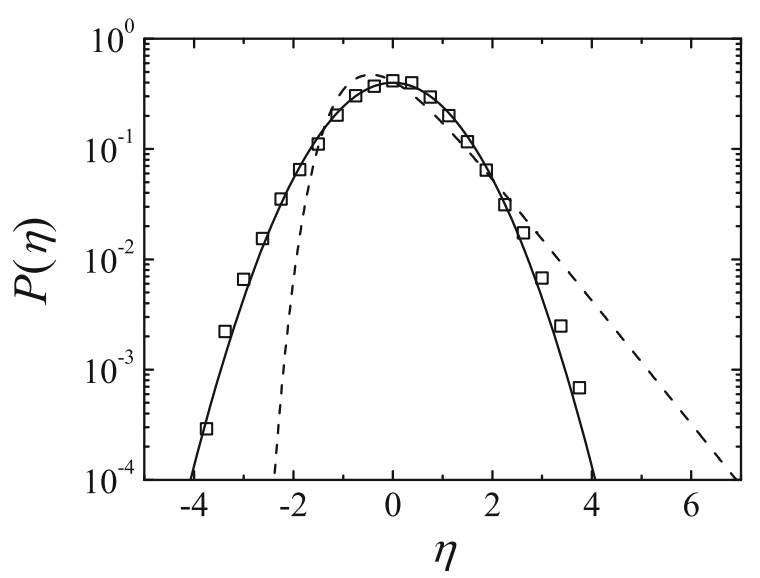

Fig. 2 Numerically calculated PDF $P(\eta)$ (open squares) as a function of the normalized net heat accumulation $\eta$ at fixed values of $\mathrm{Ra}=5 \times 10^{9}$ and $\mathrm{Pr}=4.4$. The solid line is a plot of Eq. (3). The dashed line shows a plot of Eq. (4) with $\chi=-0.001$, which is the best fit to the experimentally measured $P(\eta)$ for the data shown in Fig. 1a [75]

$J_{t}(t)$ and $J_{b}(t)$ are obtained by numerically integrating the temperature gradient at the top and bottom conducting surfaces, respectively. Unlike the experimental data shown in Fig. 1a, fluctuations of $\eta(t)$ obtained from the direct numerical simulation are symmetric and no rare massive eruption events were observed. This is clearly shown from the resulting probability density function (PDF) $P(\eta)$ of $\eta$.

Figure 2 shows a comparison between the direct numerical simulation and experimental results. It is found that the numerically obtained $P(\eta)$ (open squares) is well described by the standard Gaussian distribution function (solid line)

$P(\eta)=\frac{1}{\sqrt{2 \pi}} e^{-\eta^{2} / 2}$.

This result suggests that fluctuations of $\eta$ in the direct numerical simulation are caused primarily by independent and random emission of thermal plumes from the top and bottom conducting plates, a process to which the central limit theorem applies. As a result, the net heat flux passing through the convecting fluid is balanced almost instantaneously and there is no long-time heat accumulation, as observed in the experiment.

In contrast, the obtained PDF $P(\eta)$ from the experiment was found [75] to be highly non-Gaussian and follow the generalized extreme value distribution

$P(z)=\frac{1}{\beta}(1+\chi z)^{-(1 / \chi+1)} e^{-(1+\chi z)^{-1 / \chi}}$, where the variable $z=(\eta-\mu) / \beta$, with $\beta=$ $|\chi| /\left[\Gamma(1-2 \chi)-\Gamma^{2}(1-\chi)\right]^{1 / 2}$ and $\mu=\beta[1-\Gamma(1-$ $\chi)] / \chi$. Here $\Gamma(x)$ is the gamma function. The dashed line in Fig. 2 shows a plot of Eq. (4) with $\chi=-0.001$, which is the best fit to the experimentally measured $P(\eta)$ for the data shown in Fig. 1a [75].

\section{Theoretical model and analysis}

The above results prompt us to think what causes the disagreement between the direct numerical simulation and experimental results. In the direct numerical simulation, the Oberbeck-Boussinesq approximation [44] was used so that the entire convection system is symmetric by reflection and thus the top and bottom boundary layers are identical. In the actual experiment, however, such a symmetry is broken because of the temperature dependence of fluid properties, which is often referred to as the non-Oberbeck-Boussinesq (NOB) effect $[2,78,84]$. It was found in the recent experiment [75] that the statistical properties of the LSC cessations and reversals depend sensitively on the properties of the convecting fluid. This finding suggests that the LSC cessations and reversals might be triggered by some minute asymmetry in boundary layer dynamics owing to the NOB effects of the convecting fluid, which can cause the top and bottom boundary layers to have different thicknesses and viscosities, for instance.

At the moment, we are unable to use Nek5000 to study the NOB effect on the LSC dynamics. This is because the standard Nek5000 code is an opensource solver for incompressible Navier-Stokes equations with constant fluid properties, and the asymmetric boundary layers resulting from the NOB effect require the fluid properties to be a function of the local temperature. Numerically, the NOB effect makes the coefficients of the Poisson equation space- and timedependent, which is difficult to solve in a computationally efficient manner. To move forward, we consider the Villermaux's model [72], which consists of two coupled DDEs to describe the instabilities in the top and bottom boundary layers

$$
\begin{aligned}
& \dot{A}(t)=A(t)\left\{r-\mu_{t}\left[A\left(t-t_{0}\right)+c B\left(t-t_{0}\right)\right]\right\} \\
& \dot{B}(t)=B(t)\left\{r-\mu_{b}\left[B\left(t-t_{0}\right)+c A\left(t-t_{0}\right)\right]\right\},
\end{aligned}
$$

where $A(t)$ and $B(t)$ represent the amplitude of instabilities in the top (t) and bottom (b) boundary layers, $r$ represents their growth rate, and $c(0<c<1)$ is 
a coupling constant having a typical value of 0.5 . In the above, $\mu_{t}\left(\right.$ or $\mu_{b}$ ) is a sensitivity parameter to set the oscillation amplitude, and the delay time $t_{0}$ reflects the time interval for disturbances (thermal plumes) to travel from one boundary layer to the other.

The nonlinear dynamics as described in Eq. (5) was studied previously for a special symmetric case with $\mu_{t}=\mu_{b}[65,72]$. In this case, linear stability analysis predicts an in-phase oscillatory solution for $A(t)$ and $B(t)$, once $t_{0}$ exceeds a threshold $\left(t_{0}\right)_{c}=\pi /(2 r)$. The corresponding oscillation frequency $f_{0}$ is such that $2 \pi f_{0}=r$, leading to $f_{0}\left(t_{0}\right)_{c}=1 / 4$ in that limit. This prediction has been verified in an early convection experiment conducted in the thin disk cell [65]. While the oscillatory solution of Eq. (5) captures the primary mode of LSC, our fundamental understanding of Eq. (5) remains limited and most of its parameter space has not been explored.

We now examine Eq. (5) in a larger parameter space for the asymmetric boundary layers. As will be shown below, the long steady quiet period of the LSC is well described by the in-phase oscillatory solution, and thus, it should be treated as the primary instability of the thermal boundary layers. Two parameters, $r$ and $t_{0}$, are used to describe this common instability of the coupled boundary layers. To describe the rare but large eruptions of thermal plumes as a secondary instability of the boundary layers, we consider the NOB effect of the two boundary layers with a small difference in $\mu_{t} \neq \mu_{b}$. In this case, Eq. (5) has a fixed point

$A^{*}=\frac{r\left(\mu_{b}-c \mu_{t}\right)}{\mu_{b} \mu_{t}\left(1-c^{2}\right)}, \quad B^{*}=\frac{r\left(\mu_{t}-c \mu_{b}\right)}{\mu_{b} \mu_{t}\left(1-c^{2}\right)}$,

and the linear stability analysis near the fixed point reveals that for $t_{0}>\left(t_{0}\right)_{c}=\pi /(2 r)$, the system becomes unstable via a Hopf bifurcation, giving rise to in-phase oscillations similar to the case of $\mu_{t}=\mu_{b}$ [65]. Because both $A^{*}$ and $B^{*}$ are positive numbers, one has $c \mu_{b}<\mu_{t}<\mu_{b} / c$. Physically, $A^{*}$ and $B^{*}$ set the amplitudes (rms values) of $A(t)$ and $B(t)$. In particular, because of the NOB asymmetry, the amplitude ratio of the emerged oscillations of $A(t)$ and $B(t)$ is given by $B^{*} / A^{*}=\left(\mu_{t}-c \mu_{b}\right) /\left(\mu_{b}-c \mu_{t}\right)$.

In the experiment, we find that the peak value $\left(\sigma_{T} / \Delta T\right)_{\max }$ of the normalized temperature rms profile near the top conducting plate is always larger than that near the bottom conducting plate for all the convecting fluids used and all the Rayleigh numbers studied [73]. Figure 3 shows a sample comparison of the

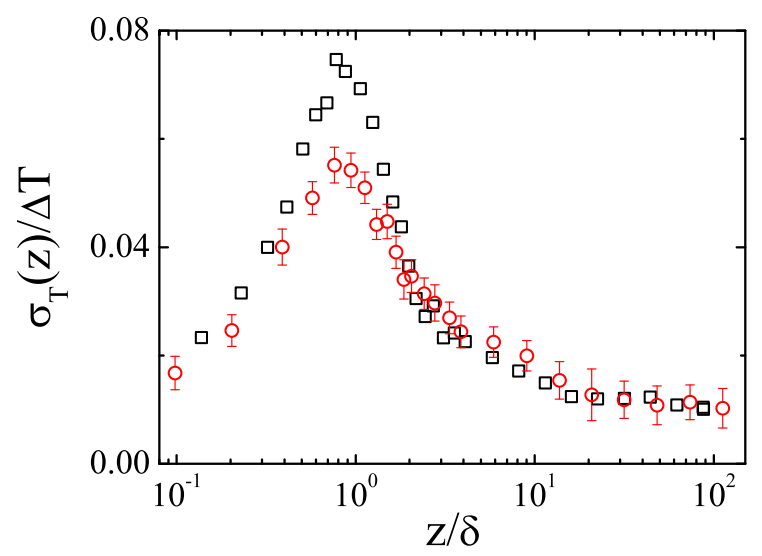

Fig. 3 Measured temperature rms profile $\sigma_{T}(z) / \Delta T$ as a function of $z / \delta$ near the bottom conducting plate (red circles) and top conducting plate (black squares). Here $\sigma_{T}(z)$ is normalized by the temperature difference $\Delta T$ between the two conducting plates, and the distance $z$ away from the conducting plate is normalized by the thickness $\delta$ of the bottom and top thermal boundary layers, respectively. The measurements are made at $\mathrm{Ra}=5.3 \times 10^{9}$ and $\operatorname{Pr}=7.6$ (20\% glycerin-aqueous solution). The error bars indicate the experimental uncertainties. (Color figure online)

measured temperature rms profiles $\sigma_{T}(z) / \Delta T$ near the bottom and top conducting plates. This result suggests that $\mu_{t}<\mu_{b}$, as the oscillation amplitude $A(t)$ [or $B(t)]$ is inversely proportional to $\mu_{t}$ (or $\mu_{b}$ ) [65].

As a time-delay system with $t_{0}>0$, Eq. (5) can be approximated by a system of ordinary differential equations with an infinite number of dimensions, in the sense that infinitely many initial conditions over the continuous range $-t_{0}<t<0$ are needed. Equation (5) thus can be written as [42]

$$
\begin{aligned}
& \dot{A}_{0}(t)=A_{0}(t)\left\{r-\mu_{t}\left[A_{N}(t)+c B_{N}(t)\right]\right\} \\
& \dot{B}_{0}(t)=B_{0}(t)\left\{r-\mu_{b}\left[B_{N}(t)+c A_{N}(t)\right]\right\} \\
& \dot{A}_{i}(t)=\frac{N}{t_{0}}\left[A_{i-1}(t)-A_{i}(t)\right] \\
& \dot{B}_{i}(t)=\frac{N}{t_{0}}\left[B_{i-1}(t)-B_{i}(t)\right] \quad 1 \leq i \leq N \rightarrow \infty .
\end{aligned}
$$

With the parameters discussed above, Eq. (5) can be numerically solved using an open-source DDE solver, XPPAUT [63]. Our numerical solutions are also verified by the numerical results of Eq. (7) with $N$ between 400 and 500 using a homemade code. Therefore, we conclude that our numerical results are reliable. In the simulation, $\mu_{t}$ and $t_{0}$ are chosen as two control parameters and the other parameters are fixed at $\mu_{b}=r=1$ and $c=0.5$. Figure 4 shows the "phase diagram" 


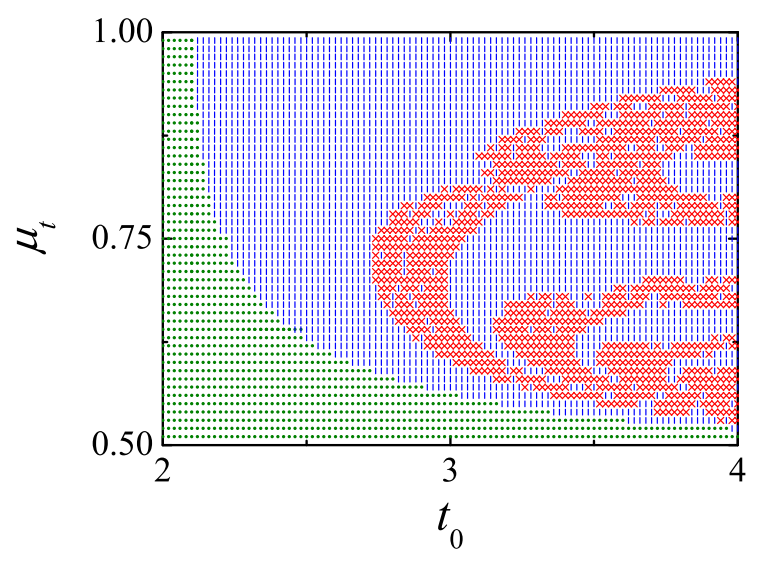

Fig. 4 Phase diagram of the solutions of Eq. (5) in the $\left(\mu_{t}\right.$, $\left.t_{0}\right)$ plane. The solutions of Eq. (5) can be categorized into three regimes: (i) in-phase single-period oscillations (green dots), (ii) multi-period oscillations (vertical blue bars), and (iii) chaos (red crosses). Here $\mu_{t}$ is in unit of $r$ and $t_{0}$ is in unit of $1 / r$. (Color figure online)

of the solutions of Eq. (5) in the $\left(\mu_{t}, t_{0}\right)$ plane with $0.5 \leq \mu_{t} \leq 1$ and $2 \leq t_{0} \leq 4$. Here $\mu_{t}$ is in unit of $r$ and $t_{0}$ is in unit of $1 / r$. The solutions of Eq. (5) can be categorized into three regimes: (i) in-phase single-period oscillations (green dots), (ii) multi-period oscillations (vertical blue bars), and (iii) chaos (red crosses). This phase diagram remains unchanged for different initial conditions.

Similar to the previously studied symmetric case [65], Eq. (5) with $\mu_{t} \neq \mu_{b}$ destabilizes as soon as $t_{0}>\pi / 2$, leading to an in-phase single-period oscillation solution for $A(t)$ and $B(t)$ (Regime (i) in Fig. 4). Figure 5 shows a representative time sequence of $A(t)$ (black line) and $B(t)$ (red line), which are obtained with $\mu_{t}=0.75$ and $t_{0}=2.1$. In this regime, when $t_{0}$ is just above the threshold $\pi / 2$, the oscillation frequency $f_{0}$ satisfies the condition $f_{0} t_{0}=1 / 4$. When $t_{0}$ is further increased, the value of $f_{0} t_{0}$ decreases gradually from $1 / 4$. From Fig. 5, we find the oscillation amplitude ratio $B / A \simeq 1.425 / 3.564 \simeq 0.4$, which agrees well with the theoretical prediction, $B^{*} / A^{*}=$ $\left(\mu_{t}-c \mu_{b}\right) /\left(\mu_{b}-c \mu_{t}\right)=0.4$.

With further increase in $t_{0}$, the system enters the multi-period oscillation regime (Regime (ii) in Fig. 4), in which the solutions of $A(t)$ and $B(t)$ have multiple periods. Figure 6 shows a representative time sequence of $A(t)$ (black line) and $B(t)$ (red line) when $t_{0}$ just enters the regime with $\mu_{t}=0.75$ and $t_{0}=2.3$. The obtained $A(t)$ and $B(t)$ both have two periods

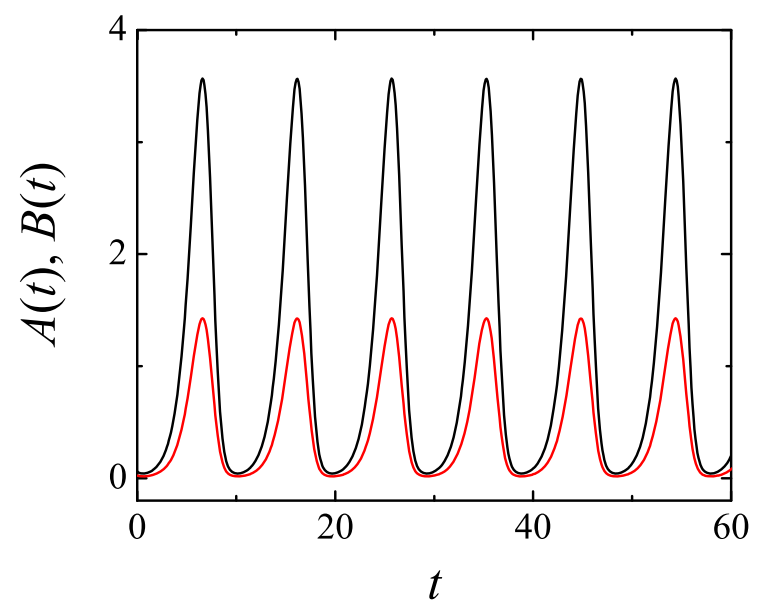

Fig. 5 Numerical solution of Eq. (5) for $A(t)$ (black line) and $B(t)$ (red line) with $\mu_{t}=0.75$ and $t_{0}=2.1$. (Color figure online)

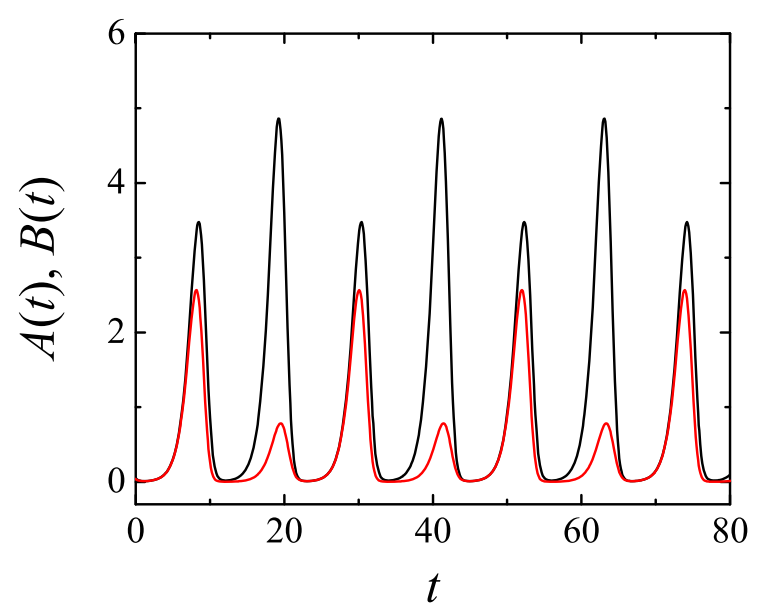

Fig. 6 Numerical solution of Eq. (5) for $A(t)$ (black line) and $B(t)$ (red line) with $\mu_{t}=0.75$ and $t_{0}=2.3$. (Color figure online)

and they compete with each other, so that the largeamplitude period in $A(t)$ always corresponds to the small-amplitude period in $B(t)$. This behavior continues for the solutions of $A(t)$ and $B(t)$ with more oscillation periods. As the value of $t_{0}$ further increases, the solutions of $A(t)$ and $B(t)$ evolve from period-2 to period-4 and so on, undergoing period-doubling and eventually become chaotic.

In the chaos regime (Regime (iii) in Fig 4), both $A(t)$ and $B(t)$ show an irregular time sequence. Figure 7 shows a representative time sequence of $A(t)$ (upper black line) and $B(t)$ (lower red line) with $\mu_{t}=0.75$ and $t_{0}=3.0$. In the plot, $A(t)(B(t))$ is normalized 

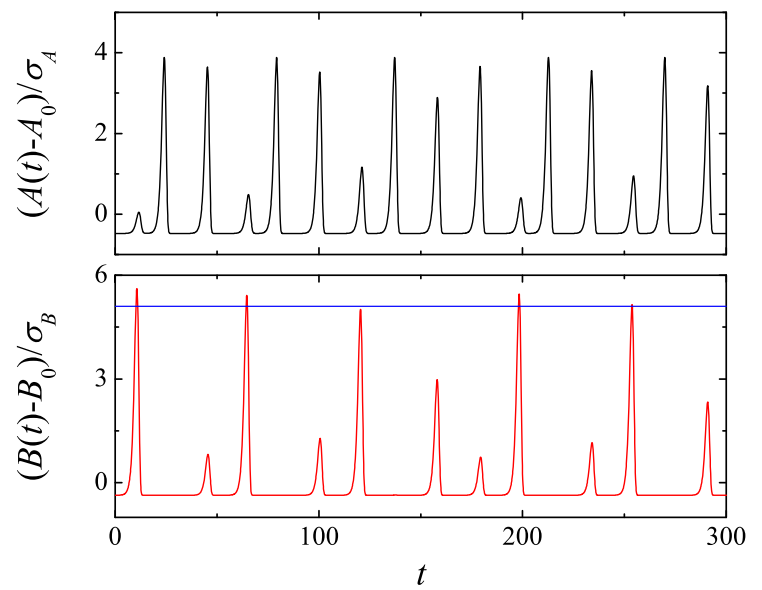

Fig. 7 Numerical solution of Eq. (5) for the normalized time series, $\left(A(t)-A_{0}\right) / \sigma_{A}$ (upper black line) and $\left(B(t)-B_{0}\right) / \sigma_{B}$ (lower red line), with $\mu_{t}=0.75$ and $t_{0}=3.0$. Here $A_{0}\left(B_{0}\right)$ and $\sigma_{A}\left(\sigma_{B}\right)$ are, respectively, the mean value and standard deviation of $A(t)(B(t))$. The horizontal blue line indicates the threshold value $\chi_{c} \equiv\left(B_{c}-B_{0}\right) / \sigma_{B}=5$, which is used to determine the eruption events with $\left(B(t)-B_{0}\right) / \sigma_{B}>\chi_{c}$. (Color figure online)

as $\left(A(t)-A_{0}\right) / \sigma_{A}\left(\left(B(t)-B_{0}\right) / \sigma_{B}\right)$, where $A_{0}\left(B_{0}\right)$ and $\sigma_{A}\left(\sigma_{B}\right)$ are, respectively, the mean value and standard deviation of $A(t)(B(t))$. While both $A(t)$ and $B(t)$ appear chaotic in amplitude, they still contain a phase-synchronized oscillatory component with its frequency being $f_{0} t_{0} \simeq 1 / 6$. This frequency is smaller than those in the other two regimes. Note that there exist small windows of multi-period oscillations in the chaos regime, which is a common feature of deterministic chaos systems [62].

We now present the solutions of $A(t)$ and $B(t)$ in a different way. Figure 8 shows the characteristic phase portraits of $B(t)$ versus $A(t)$ in the three dynamic regimes. In the plot, we fix the value of $\mu_{0}=0.75$ and vary the value of $t_{0}$ systematically. As shown in Fig. 8(a), the phase portrait for in-phase single-period oscillations is a straight line, indicating that $A(t)$ and $B(t)$ are proportional to each other. Figures $8(\mathrm{~b})$ and 8(c) show the phase portraits for multi-period oscillations, which have an even number of loops. The number of loops is doubled when the value of $t_{0}$ is increased from 2.3 to 2.7. With a further increase in $t_{0}$, the number of loops doubles and the oscillations in $A(t)$ and $B(t)$ are no longer in-phase any more. Finally, when $t_{0}=3.0$, the number of loops in the phase portrait becomes so large that the system enters the chaos regime. This is shown in Fig. 8(d). In Fig. 4, we define
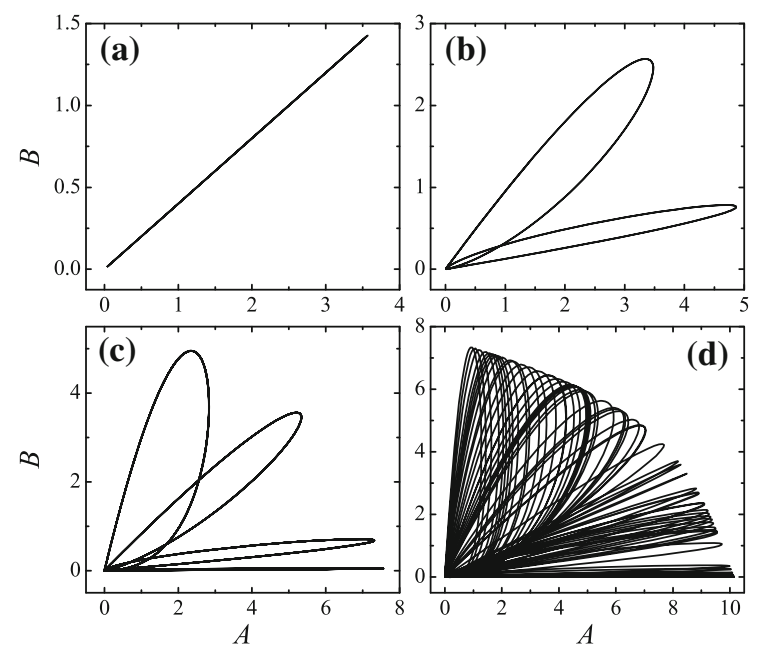

Fig. 8 Phase portraits of $B(t)$ versus $A(t)$ at a fixed value of $\mu_{0}=0.75$ and different values of $t_{0}: \mathbf{a} t_{0}=2.1$ (period-1), b $t_{0}=2.3($ period -2$), \mathbf{c} t_{0}=2.7($ period -4$)$, and $\mathbf{d} t_{0}=3.0($ chaos $)$

the multi-period oscillation regime by setting the number $\mathcal{N}$ of loops in the phase portrait to be in the range of $2 \leq \mathcal{N} \leq 50$. The chaos regimes are defined for $\mathcal{N}>50$.

To further examine the dynamic behavior of the solutions of $A(t)$ and $B(t)$ in different regimes, we compute their Lyapunov exponent using the idea of the Wolf algorithm [77]. For our purpose, only the maximal value of the Lyapunov exponent, $\lambda_{L}^{\max }$, is needed to identify the dynamics [62]: $\lambda_{L}^{\max }<0$ for no oscillation (fixed point), $\lambda_{L}^{\max } \simeq 0$ for periodic motion (limit cycle) and $\lambda_{L}^{\max }>0$ for chaos (strange attractor). Figure 9(a) and 9(b) shows the calculated $\lambda_{L}^{\max }$ as a function of $t_{0}$ at a fixed value of $\mu_{0}=0.75$. It is found $\lambda_{L}^{\max }<0$ for $t_{0}<\pi / 2$, which is in good agreement with the stability analysis discussed above. As the value of $t_{0}$ increases, we find $\lambda_{L}^{\max } \simeq 0$ for the ranges $\pi / 2 \leq t_{0}<2.75$ and $3.05 \lesssim t_{0} \lesssim 3.7$, and $\lambda_{L}^{\max }>0$ for $2.75 \lesssim t_{0}<3.05$. The calculated values of $\lambda_{L}^{\max }$ provide further support to the phase diagram depicted in Fig. 4. Note that Fig. 9(b) reveals a special point $t_{0} \simeq 2.85$, at which $\lambda_{L}^{\max } \simeq 0$. This corresponds exactly to the small window of the multi-period oscillation regime embedded in the chaos regime as shown in Fig. 4.

One can also obtain the local maximum values $B_{p}$ from the time series $B(t)$ and investigate how the statistics of $B_{p}$ evolve in different dynamic regimes. Figure 9(c) shows the bifurcation diagram of $B_{p}$ as a func- 

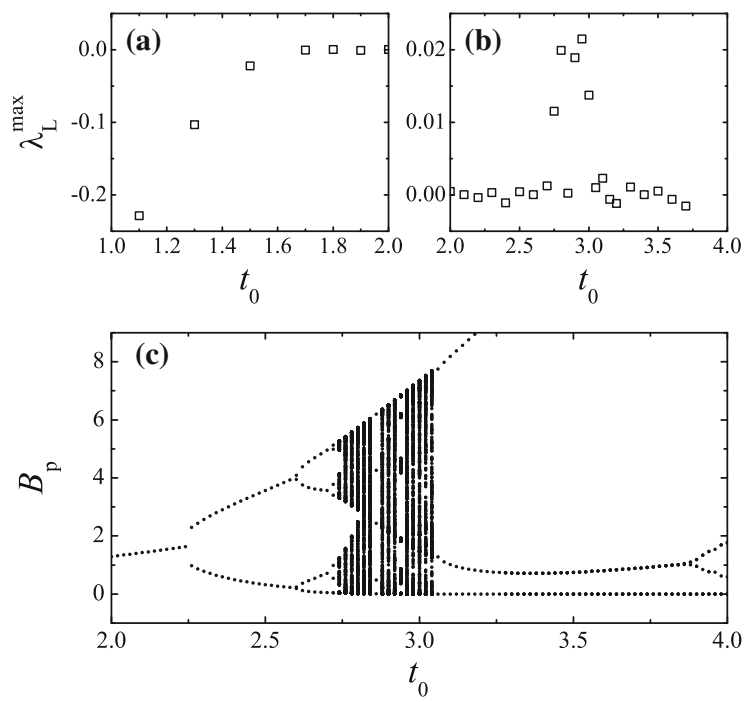

Fig. 9 Calculated maximal value of the Lyapunov exponent, $\lambda_{L}^{\max }$, as a function of $t_{0}$ in the ranges: a $1.0 \lesssim t_{0} \lesssim 2.0$ and b $2.0 \lesssim t_{0} \lesssim 4.0$. The calculation is made at a fixed value of $\mu_{0}=0.75$, c Corresponding bifurcation diagram of the local maximum values $B_{p}$ of the time series data $B(t)$ as a function of $t_{0}$

tion of $t_{0}$ at a fixed value of $\mu_{0}=0.75$. In the singleperiod oscillation regime with $\pi / 2 \lesssim t_{0} \lesssim 2.25, B_{p}$ has only one value for a given $t_{0}$, which gives rise to a single-valued curve in the bifurcation diagram. In the range of $2.25 \lesssim t_{0} \lesssim 3.04$, the number of the $B_{p}$ values doubles repeatedly with increasing $t_{0}$ and finally becomes a very large number, indicating that the period number in $B(t)$ becomes so large that the solution of $B(t)$ is chaotic. Figure 9(c) thus confirms that the system evolves toward chaos through a period-doubling route [62]. Furthermore, we find that the region of $t_{0}$ with a large number of the $B_{p}$ values, as shown in Fig. 9(c), overlaps with the region having $\lambda_{L}^{\max }>0$, as shown in Fig. 9(b). This result further confirms the existence of the chaos regime.

To compare the dynamic behavior of $A(t)$ and $B(t)$ with the experimental results, we calculate their crosscorrelation function

$$
C\left(t^{\prime}\right)=\frac{\left\langle\left(A\left(t+t^{\prime}\right)-A_{0}\right)\left(B(t)-B_{0}\right)\right\rangle}{\sigma_{A} \sigma_{B}},
$$

where $\langle\ldots\rangle$ denotes an average over time $t$. Figure 10 shows a comparison between the calculated (black line) and measured (red line) $C\left(t^{\prime}\right)$ as a function of the normalized delay time $t^{\prime} f_{0}$. It is seen that the calculated $C\left(t^{\prime}\right)$ reveals a well-defined oscillation of frequency $f_{0}$ between $A(t)$ and $B(t)$, which overlaps with the mea-

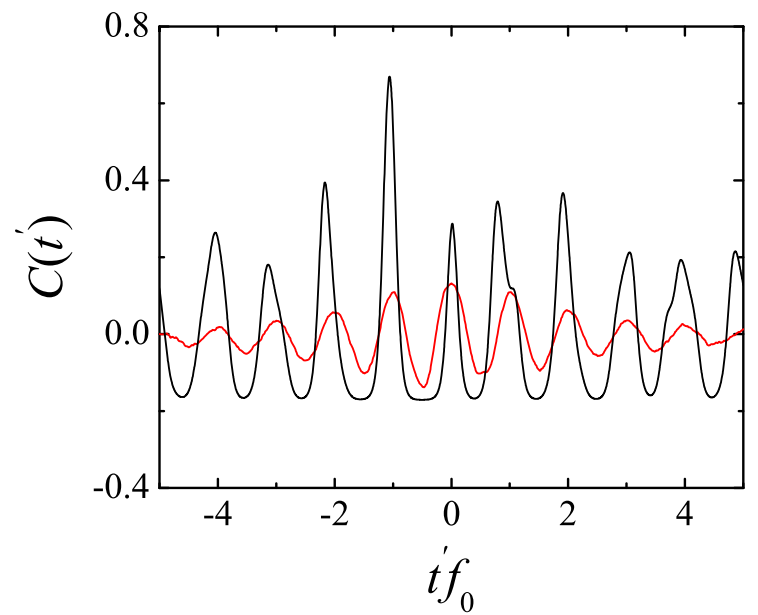

Fig. 10 Comparison between the calculated (black line) and measured (red line) cross-correlation function $C\left(t^{\prime}\right)$ as a function of the normalized delay time $t^{\prime} f_{0}$. The numerical solution of Eq. (5) is obtained with $\mu_{t}=0.75$ and $t_{0}=3.0$. The measured $C\left(t^{\prime}\right)$ is obtained from two local temperature signals in the convecting fluid: one is obtained at the mid-plane and $2 \mathrm{~cm}$ away from the sidewall of the thin disk cell and the other is obtained from the opposing sidewall of the thin disk. The local temperature measurements are made at $\mathrm{Ra}=1.1 \times 10^{10}$ and $\mathrm{Pr}=7.6$ (20\% glycerin-aqueous solution). Because temperature fluctuations produced by warm plumes at one position and by cold plumes at the opposing position have a sign difference [65], a correction of sign is introduced in the measured $C\left(t^{\prime}\right)$. (Color figure online)

sured $C\left(t^{\prime}\right)$ for temperature fluctuations at two opposite locations in the mid-plane of the vertical disk. The experimental data contain random fluctuations resulting from the turbulent background, which is a natural source for "dephasing" (or "decoherence"). As a result, the measured $C\left(t^{\prime}\right)$ has a smaller correlation amplitude and a shorter correlation time compared with the calculated $C\left(t^{\prime}\right)$. The obtained $A(t)$ and $B(t)$ from Eq. (5), on the other hand, lack this dephasing noise even in the chaos regime, which we believe is the main reason for the long-time correlation (or non-decaying behavior) of the calculated $C\left(t^{\prime}\right)$. This type of cross-correlation functions was also observed in other spatiotemporal dynamic systems with time delays [82]. The largest value of the calculated $C\left(t^{\prime}\right)$ is located at $t^{\prime} f_{0}=-1$, instead of the origin, which is caused by the competition of the oscillation amplitudes between $A(t)$ and $B(t)$. As shown in Figs. 6 and 7, the largest amplitude in $A(t)$ is always against the smallest amplitude in $B(t)$, which seems to be a generic property of this dynamic system. 
By a careful examination of the time series data of the measured temperature fluctuations, we find that the in-phase oscillation in the measured $C\left(t^{\prime}\right)$ is associated with a simultaneous and periodic emission of cold and warm plumes from the top and bottom thermal boundary layers, respectively. This periodic emission of thermal plumes has been studied in detail in a previous experiment [65]. Figure 10 thus demonstrates the existence of a direct coupling between the top and bottom boundary layers, which is mediated by the LSC and is responsible for a global oscillation, in the absence of other possible mechanisms. It also suggests that the above calculations indeed capture the essential physics of this system.

As shown in Fig. 7, the normalized amplitude of $B(t)$ has larger fluctuations compared with that for $A(t)$. To find connections between the large-amplitude spikes in $B(t)$ and the massive eruption events observed in Fig. 1(a), we set a threshold value $\chi_{c} \equiv\left(B_{c}-\right.$ $\left.B_{0}\right) / \sigma_{B}=5$ (horizontal blue line in Fig. 7), so that all the spikes with an amplitude larger than $\chi_{c}$ are counted as eruption events. Here we use a normalized (dimensionless) threshold value $\chi_{c}$, so that it can be used consistently for different values of the control parameters $\mu_{t}$ and $t_{0}$. With this definition of eruption events, we compute the time interval $\tau$ between successive eruption events and study how its probability density function (PDF or normalized histogram) $P(\tau)$ changes with different values of $\mu_{1}$ and $t_{0}$.

Figure 11 shows the calculated $\operatorname{PDF} P(\tau) / P_{0}$ for two sets of values of $\mu_{1}$ and $t_{0}$, where $P_{0}$ is the maximum value of $P(\tau)$. It is seen that the two sets of data can all be well described by a simple exponential function, $P(\tau) / P_{0} \simeq \exp \left(-\tau / \tau_{0}\right)$, with the mean time interval $\tau_{0}$ being the only fitting parameter. The results shown in Fig. 11 are in good agreement with the experiment [75], in which the measured PDF $P(\tau)$ for the massive eruption events shows an exponential form. The same procedure is also applicable to $A(t)$ and we find that the PDF $P(\tau) / P_{0}$ for successive eruption events in $A(t)$ also has a simple exponential form (not shown). There exists a minimum value $\left(\chi_{c}\right)_{\text {min }} \simeq 5.0$ for the threshold $\chi_{c}$, and the obtained PDFs have a simple exponential form only when $\chi_{c}>\left(\chi_{c}\right)_{\text {min }}$.

To verify that the exponential PDF $P(\tau)$ shown in Fig. 11 is indeed relevant to turbulent RBC, rather than for some generic extreme events often observed in chaotic systems $[59,83]$, we examine how the fitted values of the mean time interval $\tau_{0}$ change with $\mu_{t}$ and

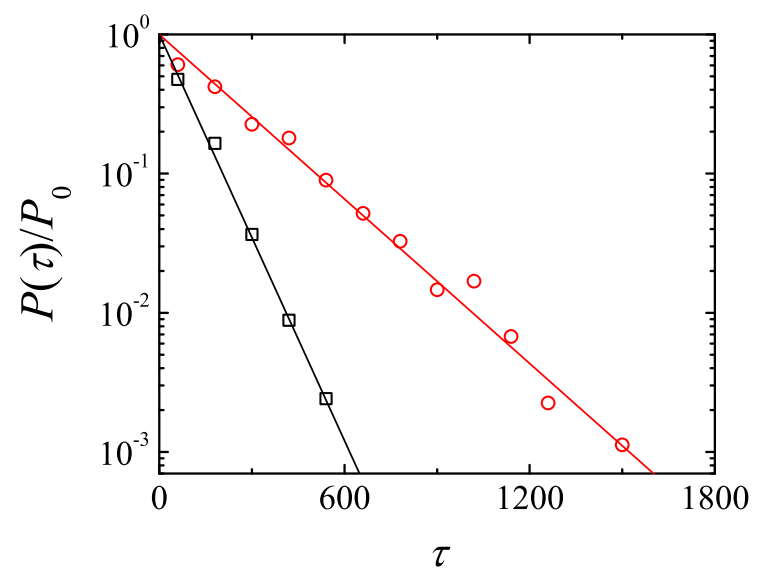

Fig. 11 Calculated PDF (or normalized histogram) $P(\tau) / P_{0}$ of the time interval $\tau$ between successive eruption events in $B(t)$. The calculations are made with $\mu_{t}=0.65, t_{0}=3.00$ (black squares) and $\mu_{t}=0.80, t_{0}=3.00$ (red circles). The colored solid lines are the exponential fits, $P(\tau) / P_{0} \simeq \exp \left(-\tau / \tau_{0}\right)$, to the data points with $\tau_{0}=89.3$ (black line) and $\tau_{0}=220.4$ (red line). (Color figure online)

$t_{0}$. Figure 12 shows the fitted values of $\tau_{0}$ as a function of $\mu_{t}$ at a fixed value of $t_{0}=3.0$ for three different values of the threshold $\chi_{c}$. It is seen that for all values of $\chi_{c}, \tau_{0}$ increases with $\mu_{t}$ and the rate of increase becomes larger for lager values of $\chi_{c}$. This result suggests that eruption events occur more frequently (i.e., with smaller values of $\tau_{0}$ ) when the top and bottom boundary layers become more asymmetric (i.e., for smaller values of $\mu_{t}$ ). This is a general trend which also holds for other values of $t_{0}$. This finding is consistent with the experimental observation that the frequency of massive eruptions of thermal plumes increases in the aqueous solutions with increasing glycerin concentration [75]. The increase in glycerin concentration enhances the NOB effect $[2,78,84]$ and so does the asymmetry between the top and bottom boundary layers. At the minimum threshold value $\left(\chi_{c}\right)_{\min } \simeq 5.0$, we find the value of $\tau_{0}$ is approximately four times of the in-phase oscillation period, i.e., $\tau_{0} f_{0} \simeq 4$. This value of $\tau_{0} f_{0}$ increases considerably with increasing $\chi_{c}$.

Figure 13 shows the fitted values of $\tau_{0}$ as a function of $t_{0}$ at a fixed value of $\mu_{t}=0.8$ for three different values of the threshold $\chi_{c}$. It is seen that for all values of $\chi_{c}, \tau_{0}$ decreases almost exponentially with increasing $t_{0}$, indicating that the eruption events occur more frequently with increasing delay time $t_{0}$ for thermal plumes to travel from one boundary layer to the other. This is a general trend which also holds for other values 


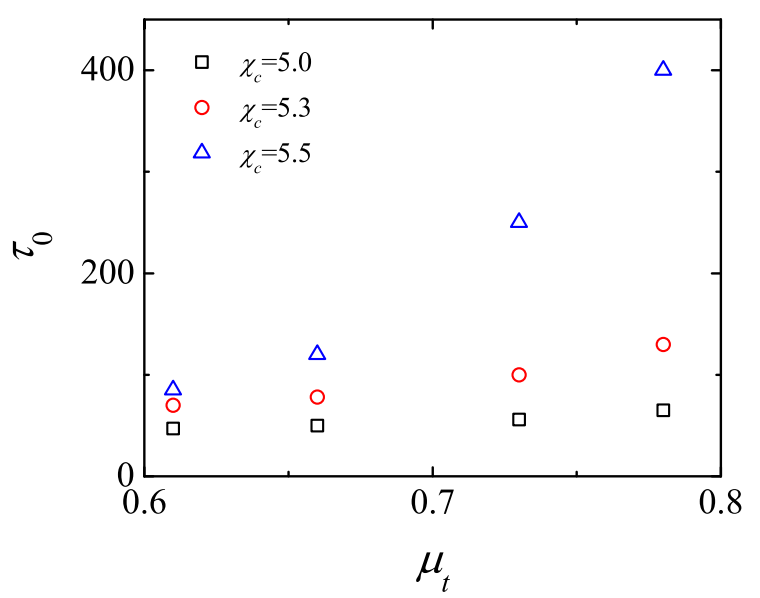

Fig. 12 Fitted values of the mean time interval $\tau_{0}$ between successive eruptions as a function of $\mu_{t}$ at a fixed value of $t_{0}=3.0$ for three different values of the threshold: $\chi_{c}=5.0$ (black squares), $\chi_{c}=5.3$ (red circles), and $\chi_{c}=5.5$ (blue triangles). (Color figure online)

of $\mu_{t}$. This finding is consistent with the experimental observation that the frequency of massive eruptions of thermal plumes decreases with increasing $\mathrm{Ra}$ [75]. In a previous experiment [65], the delay time $t_{0}$ was found to scale with the traveling time $D / U_{0}$ of the LSC across the cell, where $U_{0} \sim \mathrm{Ra}^{1 / 2}$ is the speed of the LSC. Thus, we have $t_{0} \sim \mathrm{Ra}^{-1 / 2}$, which suggests that an increase in Ra will result in a decrease of $t_{0}$. This decrease in $t_{0}$ will in turn result in an increase in $\tau_{0}$ so that the frequency of massive eruptions of thermal plumes is reduced. Our understanding of this effect is that as Ra increases, the convective flow becomes more effective in keeping the system at its primary instability state with in-phase oscillations between the two coupled boundary layers, so that the global heat transport is balanced and thus the frequency of massive eruptions is reduced.

\section{Summary}

Time delays occur ubiquitously in nature due to finite speeds of signal propagation or memory effects, and also in engineering due to processing delays. Timedelay systems can be described by relatively simple DDEs, which in general lead to a dynamical system of infinite dimensions and hence can give rise to a rich array of complex dynamical behaviors [29] and a broad range of applications. DDEs have been used

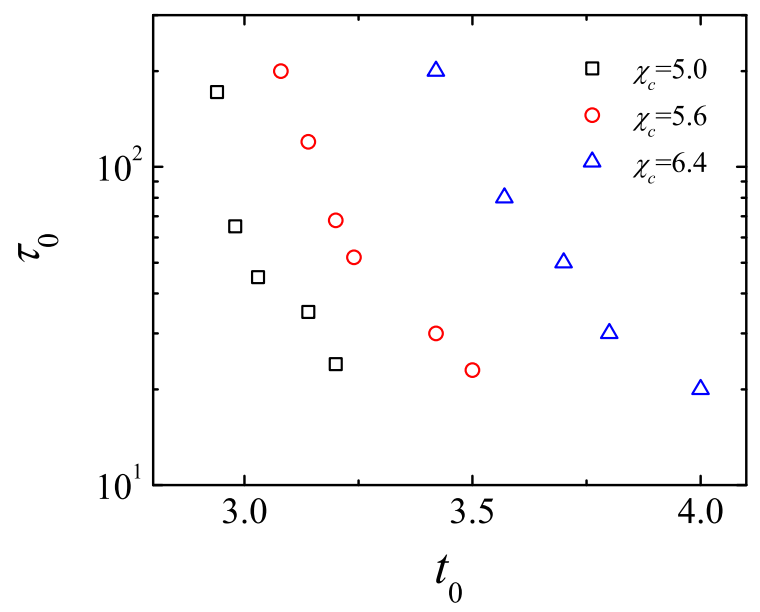

Fig. 13 Fitted values of the mean time interval $\tau_{0}$ between successive eruptions as a function of $t_{0}$ at a fixed value of $\mu_{t}=0.8$ for three different values of the threshold: $\chi_{c}=5.0$ (black squares), $\chi_{c}=5.6$ (red circles), and $\chi_{c}=6.4$ (blue triangles). (Color figure online)

to describe a number of physical phenomena involving evolution of a variable that depends not only on the current value of the variable but also on its value at time $t_{0}$ in the past. Examples include the timedelayed feedback in laser systems [46] and control of chaos in Belousov-Zhabotinsky reactions [37]. DDEs also have a broad range of applications in biological systems [58], including epidemic spreading such as the ongoing COVID-19 pandemic [4,45], propagation of action potentials between neurons [9,51], tumor growth [71], population dynamics [42,70] and respiratory model [22]. Other DDE applications in engineering and technology include electrical transmission lines [10], electrodynamics of interacting charged particles [27], machine tool vibrations [34], control of robotic machines $[40,43,49]$ and model of shimming wheels [67]. Because DDEs have an infinity number of dimensions [62], our understanding of the DDEs, and particularly their stability boundaries and corresponding routes to chaos, is often limited to a small region of parameter space.

In this work, we have carried out a systematic numerical study of two coupled nonlinear DDEs, as shown in Eq. (5). These two DDEs are a low-dimensional model for the instabilities of the top and bottom boundary layers in turbulent RBC, which are coupled by the LSC $[65,72]$. Because turbulent RBC contains many more degrees of freedom, such as those turbulent fluctuations 
as shown in Fig. 1, which are absent in the DDEs, one would not expect a one-to-one correspondence between the model calculation and actual data. Our primary goal here is to use the model to capture the essential features of the instabilities (or the "slow modes") in turbulent $\mathrm{RBC}$ with a proper physical interpretation. The nonOberbeck-Boussinesq (NOB) effect [2,78,84], which breaks the symmetry between the top and bottom boundary layers, is considered by introducing two different sensitivity parameters, $\mu_{t} \neq \mu_{b}$, in Eq. (5).

Three different types of solutions are identified and their dynamic behaviors are summarized in the phase diagram of the system as a function of the sensitivity parameter $\mu_{t}$ of the top boundary layer and the delay time $t_{0}$ for thermal plumes to travel from one boundary layer to the other. It is found that for small values of $t_{0}(\gtrsim \pi / 2 r)$, the convective flow is at a critical state near the global instability threshold, having a stable inphase oscillation between the two coupled boundary layers. The long steady quiet period observed in the convection experiments $[65,75]$ is well described by this in-phase single-period oscillatory solution, which is associated with alternate emission of thermal plumes between the two boundary layers with a local frequency $f_{0} \simeq U_{0} /(2 H)$, where $U_{0}$ is the velocity of LSC. This oscillatory solution serves as a primary instability of the thermal boundary layers, which in turn balances the global heat transport in turbulent convection.

For larger values of $t_{0}$, the system enters the multiperiod oscillation state first followed by the chaotic state. In the latter case, we find that the amplitude of $B(t)$ for the instabilities in the bottom boundary layer has large fluctuations (see Fig. 7). By a careful comparison between the numerical and experimental results, we find that the large amplitude spikes in $B(t)$ exhibit the same statistical properties as the massive eruption of thermal plumes during the short active period, as observed in the experiment. This conclusion is supported by the numerical result that the distribution of the time interval $\tau$ between successive eruption events has a simple exponential form in good agreement with the experimental finding. Furthermore, the obtained mean time interval $\tau_{0}$ between two successive eruption events is found to decrease (i.e., eruption events occur more frequently) when the top and bottom boundary layers become more asymmetric or when the delay time $t_{0}$ becomes longer. These numerical results are consistent with the experimental findings. The solutions of the two coupled nonlinear DDEs, as shown in Eq. (5), thus provide new insights into the mechanism that triggers the massive eruption of thermal plumes in turbulent $\mathrm{RBC}$

Acknowledgements This work was supported in part by RGC of Hong Kong under Grant Nos. 16301719 and N-HKUST604/19 to P.T. and by MoST of Taiwan under the Grant Nos. 107-2112M-008-003-MY3 to P.Y.L. P.Y.L. also acknowledges the support by NCTS of Taiwan.

\section{Declarations}

Conflict of interest The authors declare that they have no conflict of interest.

\section{References}

1. Ahlers, G.: Trend: turbulent convection. Physics 2, 74 (2009)

2. Ahlers, G., Brown, E., Araujo, F.F., Funfschilling, D., Grossmann, S., Lohse, D.: Non-Oberbeck-Boussinesq effects in strongly turbulent Rayleigh-Bénard convection. J. Fluid Mech. 569, 409-445 (2006)

3. Ahlers, G., Grossmann, S., Lohse, D.: Heat transfer and large scale dynamics in turbulent Rayleigh-Bénard convection. Rev. Mod. Phys. 81, 503-537 (2009)

4. Al-Darabsah, I.: Threshold dynamics of a time-delayed epidemic model for continuous imperfect-vaccine with a generalized nonmonotone incidence rate. Nonlinear Dyn. 101, 1281-1300 (2020)

5. Araujo, F.F., Grossmann, S., Lohse, D.: Wind reversals in turbulent Rayleigh-Bénard convection. Phys. Rev. Lett. 95, 084502 (2005)

6. Assaf, M., Angheluta, L., Goldenfeld, N.: Rare fluctuations and large-scale circulation cessations in turbulent convection. Phys. Rev. Lett. 107, 044502 (2011)

7. Benzi, R.: Flow reversal in a simple dynamical model of turbulence. Phys. Rev. Lett. 95, 024502 (2005)

8. Bodenschatz, E., Pesch, W., Ahlers, G.: Recent developments in Rayleigh-Bénard convection. Annu. Rev. Fluid Mech. 32, 709-778 (2000)

9. Boudkkazi, S., Carlier, E., Ankri, N., Caillard, O., Giraud, P., Fronzaroli-Molinieres, L., Debanne, D.: Release-dependent variations in synaptic latency: a putative code for short- and long-term synaptic dynamics. Neuron 56, 1048-1060 (2007)

10. Brayton, R.K.: Bifurcation of periodic solutions in a nonlinear difference-differential equations of neutral type. Q. Appl. Math. 24, 215-224 (1966)

11. Breuer, M., Hansen, U.: Turbulent convection in the zero Reynolds number limit. Europhys. Lett. 86, 24004 (2009)

12. Brown, E., Ahlers, G.: Rotations and cessations of the largescale circulation in turbulent Rayleigh-Bénard convection. J. Fluid Mech. 568, 351-386 (2006)

13. Brown, E., Ahlers, G.: A model of diffusion in a potential well for the dynamics of the large-scale circulation in turbulent Rayleigh-Bénard convection. Phys. Fluids 20, 075101 (2008) 
14. Brown, E., Ahlers, G.: Azimuthal asymmetries of the largescale circulation in turbulent Rayleigh-Bénard convection. Phys. Fluids 20, 105105 (2008)

15. Brown, E., Ahlers, G.: The origin of oscillations of the largescale circulation of turbulent Rayleigh-Bénard convection. J. Fluid Mech. 638, 383-400 (2009)

16. Busse, F.H.: Transition to turbulence in Rayleigh-Bénard convection. In: Swinney, H.L., Gollub, J. (eds.) Hydrodynamic Instabilities and the Transition to Turbulence, 2nd edn, pp. 97-137. Springer, Berlin (1985)

17. Busse, F.H.: Convection driven zonal flows and vortices in the major planets. Chaos 4, 123-134 (1994)

18. Castaing, B., Gnuaratne, G., Heslot, F., Kadanoff, L., Libchaber, A., Thomae, S., Wu, X.Z., Zaleski, S., Zanetti, G.: Scaling of hard thermal turbulence in Rayleigh-Bénard convection. J. Fluid Mech. 204, 1-30 (1989)

19. Chandra, M., Verma, M.K.: Flow reversals in turbulent convection via vertex reconnections. Phys. Rev. Lett. 110, 114503 (2013)

20. Chandrasekhar, S.: Hydrodynamic and Hydromagnetic Stability. Courier Corporation, Chelmsford (2013)

21. Cioni, S., Ciliberto, S., Sommeria, J.: Strongly turbulent Rayleigh-Bénard convection in mercury: Comparison with results at moderate Prandtl number. J. Fluid Mech. 335, 111140 (1997)

22. Cooke, K.L., Turi, J.: Stability, instability in delay equations modeling human respiration. J. Math. Biol. 32, 535543 (1994)

23. Cross, M.C., Hohenberg, P.C.: Pattern formation outside of equilibrium. Rev. Mod. Phys. 65, 851 (1993)

24. Deville, M.O., Fischer, P.F., Mund, E.H.: High-Order Methods for Incompressible Fluid Flow. Cambridge University Press, Cambridge (2002)

25. de Bruyn, J.R., Bodenschatz, E., Morris, S.W., Trainoff, S.P., Hu, Y.C., Cannell, D.S., Ahlers, G.: Apparatus for the study of Rayleigh-Bénard convection in gases under pressure. Rev. Sci. Instrum. 67, 2043-2067 (1996)

26. Drazin, P.G., Reid, W.H.: Hydrodynamic Stability. Cambridge University Press, Cambridge (2004)

27. Driver, R.D.: A neutral system with state-dependent delay. J. Differ. Equ. 54, 73-86 (1984)

28. Du, Y.B., Tong, P.: Turbulent thermal convection in a cell with ordered rough boundaries. J. Fluid Mech. 407, 57-84 (2000)

29. Erneux, T.: Applied Delay Differential Equations. Springer, New York (2009)

30. Fischer, P.F.: An overlapping Schwarz method for spectral element solution of the incompressible Navier-Stokes equations. J. Comput. Phys. 133, 84-101 (1997)

31. Funfschilling, D., Ahlers, G.: Plume motion and large-scale circulation in a cylindrical Rayleigh-Bénard cell. Phys. Rev. Lett. 92, 194502 (2004)

32. Gallet, B., Herault, J., Laroche, C., Pétrélis, F., Fauve, S.: Reversals of a large-scale field generated over a turbulent background. Geophys. Astrophys. Fluid Dyn. 106, 468-492 (2012)

33. Getling, A.V.: Rayleigh-Bénard Convection: Structures and Dynamics. World Scientific, Singapore (1998)

34. Gilsinn, D.E.: Estimating critical Hopf bifurcation parameters for a second-order delay differential equation with appli- cation to machine tool chatter. Nonlinear Dyn. 30, 103-154 (2002)

35. Glatzmaier, G.A., Coe, R.S., Hongre, L., Roberts, P.H.: The role of the Earth's mantle in controlling the frequency of geomagnetic reversals. Nature 401, 885-890 (1999)

36. Gollub, J.P., Benson, S.V.: Many routes to turbulent convection. J. Fluid Mech. 100, 449-470 (1980)

37. Guderian, A., Münster, A.F., Kraus, M., Schneider, F.W.: Electrochemical chaos control in a chemical reaction: experiment and simulation. J. Phys. Chem. A 102, 5059-5064 (1998)

38. Holman, G.D.: Solar eruptive events. Phys. Today 65, 56 (2012)

39. Howard, L.N.: Convection at high Rayleigh number. In: Goertler, H. (ed.), Applied Mechanics: Proceedings of the Eleventh International Congress of Applied Mechanics Munich (Germany), p. 1109. Springer, Berlin (1966)

40. Hu, H.Y., Wang, Z.H.: Dynamics of Controlled Mechanical Systems with Delayed Feedback. Springer, Heidelberg (2002)

41. Krishnamurti, R., Howard, L.N.: Large-scale flow generation in turbulent convection. Proc. Natl. Acad. Sci. USA 78, 1981-1985 (1981)

42. Kuang, Y.: Delay Differential Equations: with Applications in Population Dynamics. Academic Press, London (1993)

43. Keighobadi, J., Fateh, M.M., Xu, B.: Adaptive fuzzy voltage-based backstepping tracking control for uncertain robotic manipulators subject to partial state constraints and input delay. Nonlinear Dyn. 100, 2609-2634 (2020)

44. Landau, L.D., Lifshitz, E.M.: Fluid Mechanics. Pergamon, New York (1987)

45. Liu, X., Zheng, X., Balachandran, B.: COVID-19: datadriven dynamics, statistical and distributed delay models, and observations. Nonlinear Dyn. 101, 1527-1543 (2020)

46. Marino, F., Giacomelli, G.: Pseudo-spatial coherence resonance in an excitable laser with long delayed feedback. Chaos 27, 114302 (2017)

47. Maurer, J., Libchaber, A.: Rayleigh-Bénard experiment in liquid helium: frequency locking and the onset of turbulence. J. Phys. Lett. 40(16), 419-423 (1979)

48. Miesch, M., Toomre, J.: Turbulence, magnetism, and shear in stellar interiors. Annu. Rev. Fluid Mech. 41, 317-345 (2009)

49. Nayfeh, N.A., Baumann, W.T.: Nonlinear analysis of timedelay position feedback control of container cranes. Nonlinear Dyn. 53, 75-88 (2008)

50. Ni, R., Huang, S.D., Xia, K.Q.: Reversals of the largescale circulation in quasi-2D Rayleigh-Bénard convection. J. Fluid Mech. 778, R5 (2015)

51. Popovych, O.V., Yanchuk, S., Tass, P.A.: Delay- and coupling-induced firing patterns in oscillatory neural loops. Phys. Rev. Lett. 107, 228102 (2011)

52. Qiu, X.L., Tong, P.: Onset of coherent oscillations in turbulent Rayleigh-Bénard convection. Phys. Rev. Lett. 87, 094501 (2001)

53. Qiu, X.L., Tong, P.: Large-scale velocity structures in turbulent thermal convection. Phys. Rev. E 64, 036304 (2001)

54. Qiu, X.L., Tong, P.: Temperature oscillations in turbulent Rayleigh-Bénard convection. Phys. Rev. E 66, 026308 (2002) 
55. Qiu, X.L., Yao, S.H., Tong, P.: Large-scale coherent rotation and oscillation in turbulent thermal convection. Phys. Rev. E 61, R6075 (2000)

56. Resagk, C., du Puits, R., Thess, A.: Oscillations of the large scale wind in turbulent thermal convection. Phys. Fluids 18, 095105 (2006)

57. Roberts, P.H., Glatzmaier, G.A.: Geodynamo theory and simulations. Rev. Mod. Phys. 72, 1081-1123 (2000)

58. Ruan, S.: Delay differential equations in single species dynamics. In: Arino, O., et al. (eds.) Delay Differential Equations and Applications, pp. 477-517. Springer, Berlin (2006)

59. Saha, A., Feude, U.: Extreme events in FitzHugh-Nagumo oscillators coupled with two time delays. Phys. Rev. E 95, 062219 (2017)

60. Sano, M., Wu, X.Z., Libchaber, A.: Turbulence in heliumgas free convection. Phys. Rev. A 40, 6421 (1989)

61. Scheel, J.D., Emran, M.S., Schumacher, J.: Resolving the fine-scale structure in turbulent Rayleigh-Bénard convection. New J. Phys. 15, 113063 (2013)

62. Schuster, H.G., Just, W.: Deterministic Chaos: An introduction. Wiley, Hoboken (2006)

63. See. http://www.math.pitt.edu/ bard/xpp/xpp.html for more details

64. Settles, G.S.: Schlieren and Shadowgraph Techniques: Visualizing Phenomena in Transparent Media. Springer, Berlin (2012)

65. Song, H., Villermaux, E., Tong, P.: Coherent oscillations of turbulent Rayleigh-Bénard convection in a thin vertical disk. Phys. Rev. Lett. 106, 184504 (2011)

66. Sreenivasan, K.R., Bershadski, A., Niemela, J.J.: Mean wind and its reversal in thermalconvection. Phys. Rev. E 65, 056306 (2002)

67. Stépán, G.: Delay, nonlinear oscillations and shimmying wheels. In: Moon, F.C. (ed.) IUTAM Symposium on New Applications of Nonlinear and Chaotic Dynamics in Mechanics, pp. 373-386. Kluwer Academic, Dordrecht (1999)

68. Sugiyama, K., Ni, R., Stevens, R.J.A.M., Chan, T.S., Zhou, S.Q., Xi, H.D., Sun, C., Grossmann, S., Xia, K.Q., Lohse, D.: Flow reversals in thermally driven turbulence. Phys. Rev. Lett. 105, 034503 (2010)

69. Sun, C., Xia, K.Q., Tong, P.: Three-dimensional flow structures and dynamics of turbulent thermal convection in a cylindrical cell. Phys. Rev. E 72, 026302 (2005)

70. Upadhyay, R.K., Agrawal, R.: Dynamics and responses of a predator-prey system with competitive interference and time delay. Nonlinear Dyn. 83, 821-837 (2016)

71. Villasana, M., Radunskaya, A.: A delay differential equation model for tumor growth. J. Math. Biol. 47, 270-294 (2003)
72. Villermaux, E.: Memory-induced low frequency oscillations in closed convection boxes. Phys. Rev. Lett. 75, 4618 (1995)

73. Wang, Y., He, X.Z., Tong, P.: Boundary layer fluctuations and their effects on mean and variance temperature profiles in turbulent Rayleigh-Bénard convection. Phys. Rev. Fluids 1, 082301(R) (2016)

74. Wang, Y., He, X.Z., Tong, P.: Turbulent temperature fluctuations in a closed Rayleigh-Bénard convection cell. J. Fluid Mech. 874, 263-284 (2019)

75. Wang, Y., Lai, P.Y., Song, H., Tong, P.: Mechanism of largescale flow reversals in turbulent thermal convection. Sci. Adv. 4, aat7480 (2018)

76. Wang, Y., Xu, W., He, X.Z., Yik, H.F., Wang, X.P., Schumacher, J., Tong, P.: Boundary layer fluctuations in turbulent Rayleigh-Bénard convection. J. Fluid Mech. 840, 408-431 (2018)

77. Wolf, A., Swift, J.B., Swinney, H.L., Vastano, J.A.: Determining Lyapunov exponents from a time series. Physica D 16, 285-317 (1985)

78. Wu, X.Z., Libchaber, A.: Non-Boussinesq effects in free thermal convection. Phys. Rev. A 43, 2833-2839 (1991)

79. Xi, H.D., Lam, S., Xia, K.Q.: From laminar plumes to organized flows: the onset of large-scale circulation in turbulent thermal convection. J. Fluid Mech. 503, 47-56 (2004)

80. Xi, H.D., Xia, K.Q.: Cessations and reversals of the largescale circulation in turbulent thermal convection. Phys. Rev. E 75, 066307 (2007)

81. Xi, H.D., Zhou, S.Q., Zhou, Q., Chan, T.S., Xia, K.Q.: Origin of the temperature oscillation in turbulent thermal convection. Phys. Rev. Lett. 102, 044503 (2009)

82. Yanchuk, S., Giacomelli, G.: Spatio-temporal phenomena in complex systems with time delays. J. Phys. A Math. Theor. 50, 103001 (2017)

83. Zamora-Munt, J., Garbin, B., Barland, S., Massimo Giudici, M., Rios Leite, J.R., Masoller, C., Tredicce, J.R.: Rogue waves in optically injected lasers: origin, predictability, and suppression. Phys. Rev. A 87, 035802 (2013)

84. Zhang, J., Childress, S., Libchaber, A.: Non-Boussinesq effect: thermal convection with broken symmetry. Phys. Fluids 9, 1034-1042 (1997)

Publisher's Note Springer Nature remains neutral with regard to jurisdictional claims in published maps and institutional affiliations. 